\title{
Towards a more accurate annotation of tyrosine- based site-specific recombinases in bacterial genomes
}

\author{
Rob Van Houdt ${ }^{1}$, Raphael Leplae², Gipsi Lima-Mendez ${ }^{3}$, Max Mergeay ${ }^{1}$ and Ariane Toussaint ${ }^{4 *}$
}

\begin{abstract}
Background: Tyrosine-based site-specific recombinases (TBSSRs) are DNA breaking-rejoining enzymes. In bacterial genomes, they play a major role in the comings and goings of mobile genetic elements (MGEs), such as temperate phage genomes, integrated conjugative elements (ICEs) or integron cassettes. TBSSRs are also involved in the segregation of plasmids and chromosomes, the resolution of plasmid dimers and of co-integrates resulting from the replicative transposition of transposons. With the aim of improving the annotation of TBSSR genes in genomic sequences and databases, which so far is far from robust, we built a set of over 1,300 TBSSR protein sequences tagged with their genome of origin. We organized them in families to investigate: i) whether TBSSRs tend to be more conserved within than between classes of MGE types and ii) whether the (sub)families may help in understanding more about the function of TBSSRs associated in tandem or trios on plasmids and chromosomes.

Results: A total of $67 \%$ of the TBSSRs in our set are MGE type specific. We define a new class of actinobacterial transposons, related to Tn554, containing one abnormally long TBSSR and one of typical size, and we further characterize numerous TBSSRs trios present in plasmids and chromosomes of $\alpha$ - and $\beta$-proteobacteria.

Conclusions: The simple in silico procedure described here, which uses a set of reference TBSSRs from defined MGE types, could contribute to greatly improve the annotation of tyrosine-based site-specific recombinases in plasmid, (pro)phage and other integrated MGE genomes. It also reveals TBSSRs families whose distribution among bacterial taxa suggests they mediate lateral gene transfer.
\end{abstract}

\section{Background}

Tyrosine-based site-specific recombinases (TBSSRs) are well known DNA breaking-rejoining enzymes that belong to a superfamily that also includes type IB topoisomerases, including human topoisomerase I. The 3D structure and molecular mechanisms of action of several enzymes of the family are well documented [1-6].

TBSSRs are major actors in the roaming of mobile genetic elements (MGEs) in bacterial genomes. Very often called "phage-like integrases" because they were originally discovered on temperate phages (for example, $\lambda$, P2 and P22). TBSSRs do, however, i) occur on other types of MGEs and ii) catalyze various biological processes. These include the integration of temperate phage

\footnotetext{
* Correspondence: ariane.toussaint@ulb.ac.be

${ }^{4}$ Laboratoire Bioinformatique des Génomes et Réseaux (BiGRe), Université

Libre de Bruxelles, Bvd du Triomphe, Bruxelles 1050, Belgium

Full list of author information is available at the end of the article
}

genomes to become prophages and of integrated conjugative elements (ICEs), their excision at the onset of lytic growth or conjugative transfer, the integration and excision of integron cassettes, the correct segregation of plasmids and chromosomes (reviewed in [7-9]) by resolution of dimers (or higher level multimers), the resolution of cointegrates resulting from the replicative transposition of some types of transposons [10], and the excision of specific DNA fragments responsible for the transient inactivation of genes (for a general review see [11]).

In the present genomic era, TBSSR annotation is far from homogenous, whether for genomes or in databases. Misinterpretation arises from the TBSSR property of catalyzing integration/excision reactions, which are also catalyzed by two other very different types of enzymes, the serine-based site specific recombinases (SBSSRs) and the DDE transposases, the latter being closely related to

\section{Biomed Central}


retroviral integrases, with which they share the conserved aspartate-glutamate-aspartate (DDE) catalytic residues.

Despite their abundance in prokaryotic genomes, including in plasmids where they appear as one of the largest conserved protein families ([12] and Additional file 1: Table S1), TBSSRs have not been so far extensively analyzed in terms of their relative sequence conservation among various types of MGEs or chromosomes. Boyd et al. [13] showed that TBSSRs encoded by genomic islands (GIs) inserted near a tRNA locus are phylogenetically closer than they are to phage encoded ones. Similarly, Ryan et al. [14] showed that Tn4371-like ICE TBSSRs are very similar and can be easily differentiated from phage ones. However, the sets of phage proteins used in those studies were small.

In this study, using a set of over 1,300 TBSSR protein sequences tagged with their genome of origin, we attempt to investigate: i) whether TBSSRs tend to be more conserved within than between classes of MGE types, that is, whether (sub)families of TBSSRs are specific to one (sub)type of MGE and ii) whether these (sub) families may help in understanding more about the function of the plasmid encoded TBSSRs. It is indeed striking that the sole Cupriavidus eutrophus $\mathrm{H} 16$ plasmid pHG1 is predicted to encode 22 TBSSRs of 280 or more amino acids (aa) http://aclame.ulb.ac.be/perl/Aclame/Genomes/ prot_view.cgi?view=genome\&id=mge:823). A rapid count of the number of TBSSRs in plasmids suggests that it far exceeds the number of proteins closely related to known plasmid dimer resolution enzymes (for example, Cre of prophage P1 [15]) or associated with integrons previously known in plasmids [16].

We carried out a clustering analysis of 1,309 TBSSRs encoded by plasmids, phages, predicted prophages and conjugative transposons $\left(\operatorname{ICET}_{n 4371}[14,17]\right)$, Recombinases In Trio (RIT) and Bipartite Module (BIM) elements [18] and GIs. The protein sequences in each cluster/family were aligned to look for the presence of a possible catalytic domain. Each family was analyzed to determine whether TBSSR protein families were MGE type specific and to further investigate the plasmid encoded TBSSRs.

\section{Results}

A set of 1,309 TBSSR protein sequences was assembled as described in Methods (Additional file 1: Table S2). Phage, plasmid and predicted prophage encoded proteins were retrieved from the ACLAME database, and GI, $\mathrm{ICE}_{\mathrm{Tn} 4371}$, RIT and BIM proteins from previously described sets of TBSSRs [13,18-20]. Far from being an exhaustive compilation of TBSSRs annotated in available sequenced genomes, this set has the advantage that each sequence can be easily traced to its associated genetic entity. Protein sequences were compared all vs. all and clustered using a combination of the SSEARCH and MCL algorithms (see Methods for details). This produced 102 families of TBSSR proteins, called Famint (for FAMily and INTegrase, Famint 0 to 44 and 46 to 102, Sup_Tables, Famint45 not being TBSSRs). Figure 1 summarizes the size and composition of the families consisting of 4 or more proteins (56 in total). Thirteen families with 3 proteins, 13 with 2 proteins and 21 singletons (including the conjugative transposon Tn916 TBSSR, 3 TBSSRs coded by phages/viruses, 8 by predicted prophages and 10 by plasmids) will not be considered further, unless they contain proteins associated with proteins in larger families.

It is readily apparent from Table 1 that there is a good overall separation between enzymes associated with various types of MGEs. Aside from a few exceptions, TBSSRs associated with chromosomal islands, plasmids or phage and prophages fall into distinct families. The TBSSR canonical catalytic motif is located in the C-terminal part of the protein and consists of a tyrosine residue $(\mathrm{Y})$ separated by around 30 residues from an upstream arginine

(R) followed by the residues required for the activation of the catalytic Y (for a review see [21]). Despite a variable degree of identity between the proteins within a family, the multiple alignments (accessible at http:// aclame.ulb.ac.be/Resources/TBSSR/index.html) reveal a very well conserved (often $100 \%$ conservation) $\mathrm{Y}$ residue near the $\mathrm{C}$-terminal end in almost all families, separated by around 30 residues from a conserved $\mathrm{R}$ (see Table 1), pointing towards the potential catalytic motif.

\section{Mixed families}

The largest family, Famint0 (210 members), includes all but one of the GI proteins in the analyzed set. It also contains some proteins encoded by phages, predicted prophages and plasmids from the ACLAME family:vir:2, family:proph:2 and family:plasmids:226, respectively (see details in Table 1). Interestingly, TBSSRs from satellite phage P4, the so-called CP4-like islands [22] and phages F116, Sf6 and HK620 that have been reported to be similar to GI integrases [23] are part of this family. While all the GIs considered are located near a tRNA gene [13], this is the case for only 14 out of the 38 predicted prophages in this family (data not shown but accessible through http://aclame.ulb.ac.be/Tools/Prophinder/). Overall, proteins in Famint0 are not very well conserved. The family appears as a typical example of a large cluster generated by an automated procedure over a large dataset. Some sequences pull in relatively distantly related sequences, which in turn trigger the same effect, generating a pool of sequences most of which are related only through intermediates. This may be the reason for the absence of a recognizable conserved putative $\mathrm{C}$-terminal 


\section{Family size}

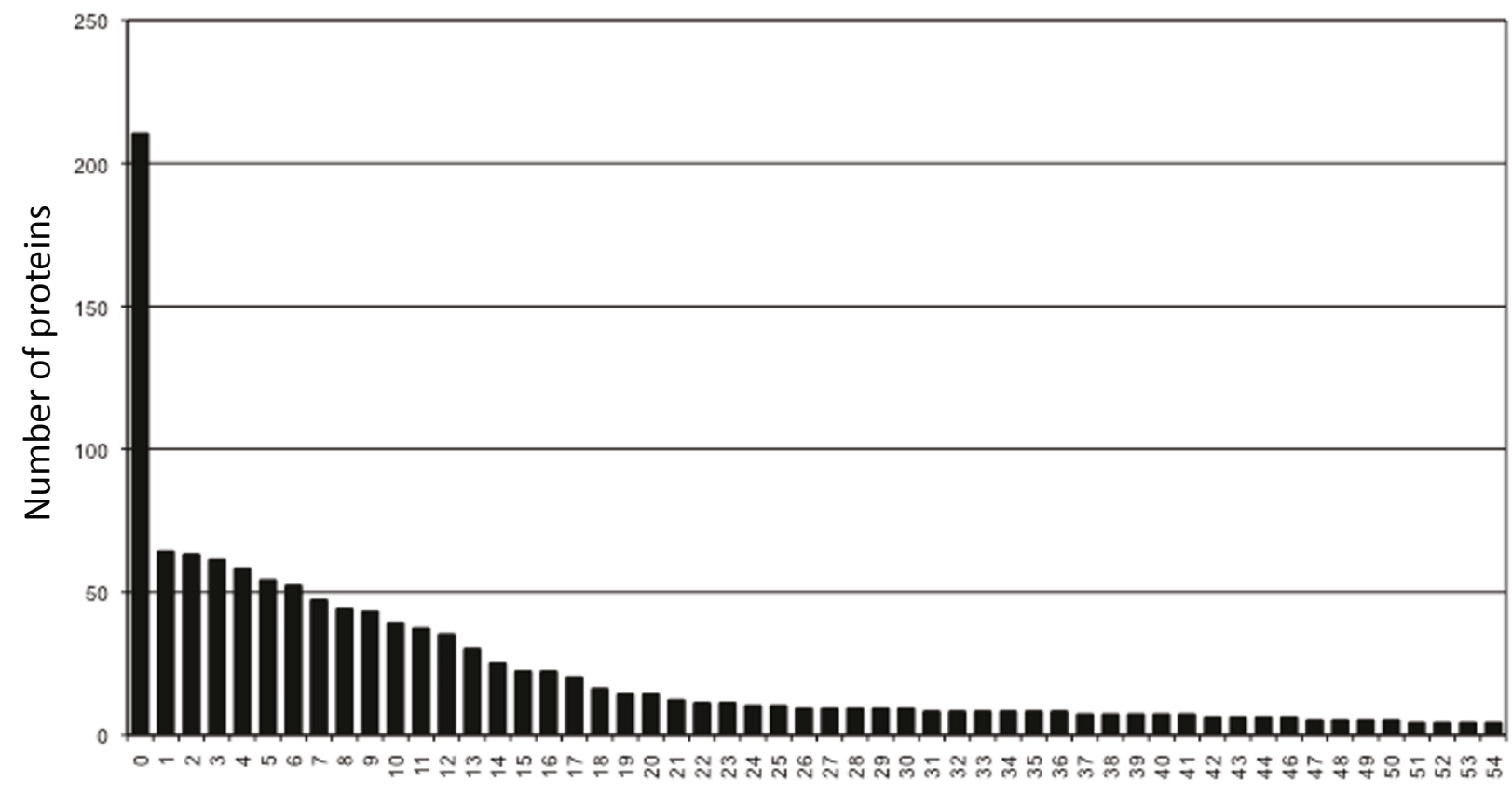

Famint ID

Figure 1 Size distribution of TBSSR families. Size distribution of the Famint families generated by MCL clustering at IF $=1.8$ and E-value 0.01 .

catalytic tetrad in the multiple sequence alignment. Alternatively, GI enzymes may be non-functional due to a long-term selection for the preservation of the island.

Besides Famint0, only six other families (Famint3, 4, $14,20,28$ and 48) are mixed and contain several proteins originating from at least two MGE types (plasmid, phage or prophage) (Table 1).

Famint3 is restricted to Firmicutes. The biological process performed by these plasmid proteins is hypothetical but since these plasmids are small, it could be the resolution of multimeric forms.

Famint14 contains TBSSRs encoded by a particular type of GIs, the conjugative transposons (or ICE) of the $\mathrm{ICE}_{\text {Tn4371 }}$ family $[14,17,20]$. In the multiple alignment, they form a clear subgroup of very conserved sequences aside from plasmid and predicted prophage proteins, the latter of which do not appear as bona fide prophages (data not shown). In this family, no obvious closer relationship exists among proteins originating from more related hosts (data not shown).

Famint20 includes TBSSRs encoded by the shufflon elements present on conjugative plasmids R64 [24], R721 [25] and Collb-P9 [26] and which, by inverting DNA segments, control the plasmids recipient specificity during mating in liquid media. The shufflon multiple inversion system consists of the TBSSR coding gene and several invertible DNA segments containing partial pilV genes separated by recombination sites. Recombination between any two inverted sites promotes the inversion of DNA segments independently or in groups, leading to the construction of several pilV genes with a constant $\mathrm{N}$-terminal but different $\mathrm{C}$-terminal segments. The resulting PilV products are adhesins located at the tip of the plasmid encoded type IV pilus, which recognizes lipopolysaccharides on the recipient cell (plasmid $153 \mathrm{kB}$ from Yersinia pseudotuberculosis IP 31758 has a single pilV gene next to the TBSSR gene). None of the nine predicted prophages contributing to Famint 20 bears or flanks a shufflon-like structure. Instead, they contain genuine phage-like genes and their TBSSR, despite being in most cases annotated "shufflon-specific DNA recombinase", appears to belong to a full or incomplete prophage. In addition, while the plasmids contributing to the family are hosted by $\gamma$-proteobacteria, the predicted prophages are in $\beta$-proteobacteria.

Famint28 includes proteins from plasmids and low score predicted prophages with no genuine phage characteristics besides replication. Only one Desulfovibrio desulfuricans predicted prophage has all expected features for being a functional prophage.

Overall, 400 proteins, that is, $30 \%$ proteins in the set do not group into MGE specific families. 
Table 1 TBSSR family analysis

\begin{tabular}{|c|c|c|c|c|c|c|c|}
\hline Famint ID & MGE type & No. prot & No. GI & No. phages & No. prophages & No. plasmids & Putative catalytic motif \\
\hline 0 & $\operatorname{int} G$ & 210 & 153 & $10($ vir2) & 38(pro2) & 9(plas226) & no obvious one \\
\hline 1 & $\operatorname{RIT}(A)$ & $64^{(4)}$ & ND & 0 & 3 & 33(plas10) & $\mathrm{RH}-\mathrm{Y}$ \\
\hline 2 & $\operatorname{RIT}(\mathrm{C})$ & $63^{(4)}$ & ND & 0 & 0 & 34(plas10) & $\mathrm{RH}-\mathrm{Y}$ \\
\hline 3 & mix & 61 & 0 & $5($ vir2) & 15(fam202) & 41 (plas170) & RK-Y \\
\hline 4 & $\operatorname{mix}$ & 58 & 0 & 27 (vir2) & 29(pro2) & 2(plas10) & $\mathrm{RH}-\mathrm{Y}$ \\
\hline 5 & $\mathrm{RIT}(\mathrm{B})$ & $54^{(4)}$ & ND & 0 & 0 & 27(plas10) & $\mathrm{RH}-Y^{(1)}$ \\
\hline 6 & (pro)phage & 52 & 0 & 16(vir2) & 36(pro2) & 0 & $\mathrm{RH} / \mathrm{R}-\mathrm{Y}$ \\
\hline 7 & (pro)phage & 47 & 0 & 18(vir2) & 29(pro2) & 0 & $\mathrm{RHT} / \mathrm{S}-\mathrm{Y}$ \\
\hline 8 & Intl & 44 & 0 & 0 & 1 (pro2) & 43(plas10) & $\mathrm{RH}-\mathrm{Y}$ \\
\hline 9 & plasmid & 43 & 0 & 0 & 0 & 43(plas10) & RHS-Y $Y^{(1)}$ \\
\hline 10 & plasmid & 39 & 0 & 0 & 0 & 39(plas10) & $\mathrm{RH}-\mathrm{Y}$ \\
\hline 11 & plasmid & 37 & 0 & 0 & 0 & 37(plas101) & $R-Y$ \\
\hline 12 & (pro)phage & 35 & 0 & 8(vir2) & 27(pro2) & 0 & RHT-Y \\
\hline 13 & (pro)phage & 30 & 0 & 11 (vir2) & 19(pro2) & 0 & $\mathrm{RH}-\mathrm{Y}$ \\
\hline 14 & $\operatorname{Tn} 4371^{(4)}$ & 25 & 13 & 0 & $5($ pro2) & 7(plas226) & $\mathrm{RH}-\mathrm{Y}$ \\
\hline 15 & (pro)phage & 22 & 0 & $3(418)$ & 19(pro76) & 0 & RSL or RLY-Y \\
\hline 16 & (pro)phage & 22 & 1 & 12(vir2) & 9(pro2) & 0 & RHT-Y \\
\hline 17 & (pro)phage & 20 & 0 & 13(vir2) & 7 (pro2) & 0 & RHS-Y \\
\hline 18 & plasmid & 16 & 0 & 0 & 0 & 16(plas101) & RSG-Y \\
\hline 19 & $\operatorname{BIM}(A)^{(4)}$ & 14 & ND & 0 & 0 & 8(plas10) & $\mathrm{RH}-\mathrm{Y}$ \\
\hline 20 & mix & 14 & 0 & 1 (vir2) & 9 (pro2) & 4(plas226) & $R-Y$ \\
\hline 21 & prophage, plasmid & 12 & 0 & 0 & $3($ pro2) & 9(plas226) & RRT-Y \\
\hline 22 & plasmid & 11 & 0 & 0 & 0 & 11 (plas10) & RRTF-Y \\
\hline 23 & plasmid & 11 & 0 & 0 & 0 & 11 (plas10) & $R-Y$ \\
\hline 24 & prophage & 10 & 0 & 0 & 10(pro2) & 0 & RK-Y \\
\hline 25 & (pro)phage & 10 & 0 & 4(vir2) & $6($ pro2) & 0 & $\mathrm{RH}-\mathrm{Y}$ \\
\hline 26 & (pro)phage & 9 & 0 & 3(vir2) & 6 (pro2) & 0 & $\mathrm{RH}-\mathrm{Y}$ \\
\hline 27 & plasmid $^{(5)}$ & 9 & 0 & 0 & 0 & 9 (plas454) & $\mathrm{RHT}-\mathrm{Y}^{(2)}$ \\
\hline 28 & phage, plasmid & 9 & 0 & 0 & 4(pro2) & 5(plas10) & $\mathrm{RH}-\mathrm{Y}$ \\
\hline 29 & mix & 9 & 0 & 1 (vir418) & 7(pro76) & 1 (plas226) & $R-Y$ \\
\hline 30 & plasmid & 9 & 0 & 0 & 0 & 9(plas688) & $R-Y$ \\
\hline 31 & plasmid & 8 & 0 & 0 & 0 & 8(plas10) & $\mathrm{RH}-\mathrm{Y}$ \\
\hline 32 & plasmid & 8 & 0 & 0 & 0 & 8(plas589) & $R-Y$ \\
\hline 33 & plasmid & 8 & 0 & 0 & 0 & 8(plas10) & $R-Y^{(2)}$ \\
\hline 34 & plasmid & 8 & 0 & 0 & 0 & 8(plas10) & RAT-Y \\
\hline 35 & (pro)phage & 8 & 0 & 1 (vir418) & 7(pro76) & 0 & No $R$ at expected distance from $Y$ \\
\hline 36 & plasmid & 8 & 0 & 0 & 0 & 8(plas10) & $\mathrm{RH}-\mathrm{Y}$, partner 41,90 \\
\hline 37 & $\operatorname{mix}$ & 7 & 0 & 0 & $6($ pro2) & 1 (plas10) & $\mathrm{RH}-\mathrm{Y}$ \\
\hline 38 & plasmid & 7 & 0 & 0 & 0 & 7 (plas101) & RR-Y \\
\hline 39 & plasmid & 7 & 0 & 0 & 0 & 7(plas589) & $\mathrm{RH}-Y^{(3)}$ \\
\hline 40 & plasmid & 7 & 0 & 0 & 0 & 7(plas10) & RHS-Y \\
\hline 41 & plasmid & 7 & 0 & 0 & 0 & 7 (plas454) & $R R-Y$ \\
\hline 42 & mix & 6 & 0 & 0 & 1 (pro2) & 5(plas10) & $\mathrm{RH}-\mathrm{Y}^{(2)}$ \\
\hline 43 & plasmid & 6 & 0 & 0 & 0 & 6(plas10) & $R R-Y^{(2)}$ \\
\hline 44 & plasmid & 6 & 0 & 0 & 0 & 6(plas10) & $\mathrm{RH}-\mathrm{Y}$ \\
\hline 46 & plasmid & 6 & 0 & 0 & 0 & 6(plas10) & RHT-Y \\
\hline 47 & $\operatorname{mix}$ & 5 & 0 & 3(vir2) & 1 (pro2) & 1 (plas226) & $\mathrm{RH}-\mathrm{Y}$ \\
\hline 48 & mix & 5 & 0 & 3(vir2) & 0 & 2(plas226) & R-Y \\
\hline 49 & plasmid & 5 & 0 & 0 & 0 & 5(plas10) & RRTAL-Y \\
\hline 50 & (pro)phage & 5 & 0 & 4(vir2) & 1 (pro2) & 0 & RHT-Y \\
\hline 51 & (pro)phage & 4 & 0 & 3(vir2) & 1 (pro2) & 0 & RHT-Y \\
\hline
\end{tabular}


Table 1 TBSSR family analysis (Continued)

\begin{tabular}{|c|c|c|c|c|c|c|c|}
\hline 52 & prophage & 4 & 0 & 0 & 4(pro76) & 0 & $R K-Y(2)$ \\
\hline 53 & plasmid $^{(5)}$ & 4 & 0 & 0 & 0 & 4(plas454) & $\mathrm{RH}-\mathrm{Y}$, partner 62 , one has no partner \\
\hline 54 & plasmid & 4 & 0 & 0 & 0 & 4(plas688) & RHTF-Y \\
\hline 55 & plasmid & 4 & 0 & 0 & 0 & 4(plas170) & R-Y \\
\hline $57-68$ & & 3 & & & & & \\
\hline $69-81$ & & 2 & & & & & \\
\hline $82-102$ & & 1 & & & & & \\
\hline
\end{tabular}

The origins of the proteins can be traced by "vir", "plas" and "pro", which stand for ACLAME family IDs (vir2 is family:vir:2, plas10 is family:plasmids:10, pro2 is family: proph:2, and so on).

(pro)phage: the family contains proteins from both phages and prophages.

Only the most conserved $\mathrm{R}$ and adjacent amino-acids and the potential catalytic $\mathrm{Y}$ residue are mentioned.

(1) Not all proteins in the family have the conserved residues.

(2) A putative catalytic motif is discernible when two shorter sequences are removed from the alignment.

(3) Distance between $\mathrm{RH}$ and $\mathrm{Y}$ is around 50 residues.

(4) Some proteins in the family do not originate from ACLAME protein families, which is the reason why the number of Gl, phages, prophages and plasmids do not add up to the number of proteins.

(5) Proteins in the family have a long N-terminal extension and are over 700 amino-acids long.

ND: Some proteins in the family could be part of a GI.

\section{Plasmid resolvases?}

The P1 Cre resolvase, a TBSSR expressed by the E. coli P1 circular plasmid prophage, is among the best structurally and biochemically characterized TBSSRs [27]. Upon clustering of phage, predicted prophage and plasmid proteins in ACLAME (version 0.4), P1 Cre joins with plasmid proteins in family:plasmids:101, pointing to the possibility that these proteins are plasmid dimer resolution enzymes. However, in the present analysis, P1 Cre belongs to a small family of only three proteins (Famint58), making this assumption shaky. ACLAME family:plasmids:101 splits here into Famint11 (which contains 37 plasmid proteins, 5 with less than 200 aa and, hence, most likely defective, and 32 of over 300 aa) and Famint18 with 16 plasmid proteins. Famint11 proteins belong to plasmids from very different hosts and several contribute two proteins to the family. Proteins in the pairs are not identical but more closely related than they are to the rest of the family members (data not shown). Famint18 contains proteins from plasmids residing in plant-interacting bacteria (except for Nitrobacter hamburgensis X14 plasmid 2). One pSymA plasmid contributes two proteins to the family. Putative catalytic sites derived from multiple alignments of Famint11, 18 and 58 members, respectively, are not the same. The present analysis thus brings no further support to a plasmid resolution function.

\section{Integrons}

Integron-encoded integrases IntI are in Famint8. The 25 IntI proteins, associated with one to eight cassettes, are almost identical at the nucleotide level. Almost all of them have been described earlier (see the plasmid names and hosts in Additional file 1: Table S1). Integrons are often associated with IS elements or transposons ( $\mathrm{Tn}$ ) that ensure their horizontal spreading (see [16]). Hence, we expected at least some IS and Tn to tend to remain associated on different plasmids. This can be readily evaluated using pre-compiled Evolutionary Conserved Modules (ECM), that is, sets of genes with similar phylogenetic profiles [28] available in the ACLAME database for different similarity thresholds (sig). IntI proteins belong to the ACLAME family:plasmids:10, which is part of ECM9, sig10. ECM9 includes, among other protein families, Tn3like transposases, SSSRs (resolvases) and IS6 transposases. This reflects the frequent association of integrons with either Tn21-like (Tn3-related) transposons, which encode these two types of proteins [29], or composite transposons, including two copies of IS6 (Tn1548 in pCTX_M3 and others) [30]. This grouping most likely results from the huge selective pressure imposed on bacterial populations by the overuse and release of antibiotics. It will be interesting to see whether these associations remain significant when more plasmid sequences of more various origins will be available. The association of integrons with Tn402 and related transposons [31], typified by the presence of the tniA-tniB and sometimes tniQ genes, appears weaker since these genes are not in ECM9 but form ECM45, with mercury resistance genes (although these also occur in integrons of Tn3-related transposons). Most other integron cassettes are in ECM13, reflecting their tendency to remain associated. Together ECM9 and 13 support the association of integrons with transposons and cohesion of the integron cassettes.

\section{BIM elements}

Famint19 regroups nine TBSSRs from $\beta$-proteobacterial hosts. Members that were originally pointed out during the annotation of the $C$. metallidurans $\mathrm{CH} 34$ genome are associated with a second conserved protein of unknown function (Famint45) making up the bipartite 
module [18]. The NCBI Protein Clusters were used to have a more complete view of these two-genes associations (Additional file 1: Table S3); however, the number of strains harboring these modules remains too low to draw any conclusion about the exact nature of this association.

\section{TBSSR combinations}

\section{Tn554-related TBSSRs}

The ACLAME family:plasmids:454 contains 20 abnormally long TBSSRs of 611 to 828 aa. Most originate from plasmids hosted by Actinobacteria. With the clustering procedure used here, the 20 proteins split into three smaller families of 9 (Famint27), 7 (Famint41) and 4 (Famint53) members, respectively. Most of these are associated with a second, adjacent and shorter TBSSR (around 350 aa) originally in the ACLAME family:plasmids:10 and here in Famint33 (partner of Famint27), Famint36 (partner of Famint41) and Famint62 (partner of Famint53). In one case, the two partners belong to Famint53 and 33, respectively. This couple resides on a Bacillus cereus plasmid and it is the only case, together with the $\alpha$-proteobacterium Novosphingobium aromaticivorans, where the host is not an Actinobacterium.

The genes corresponding to most of the couples whose members belong to Famint 27 and 33 and Famint53 and 62, are transcribed in the same direction and are associated with a third gene/protein, also similarly oriented. These third partners are found in ACLAME family:plasmids:1417 and are related to the TnpC protein of Tn554 from Staphylococcus aureus [32]. Consistent with this, Famint33, 36 and 62 proteins share significant similarity with Tn554 TnpB and Famint27 and Famint53 partners with Tn554 TnpA (data not shown). The Famint41 proteins are less related to Tn554 TnpB and have no obvious TnpC partner. Sets of contiguous genes corresponding to proteins in the same family align at the nucleotide level and these sequences can also be found in chromosomes of other Actinobacteria (Mycobacterium, Streptomyces, Rhodococcus, Table 2). The NCBI Protein Clusters provide a direct view of these sets of contiguous related clusters, which fit well with the Famint for the genomes common to the two data sets (Table 2).

Tn554 has a unique integration site [33]. Some of the genomes that carry the elements discussed here have two or more identical copies of the same $\operatorname{tn} p A B(C)$ association (for example, Mycobacterium vanbaalenii PYR-1 chromosome, Streptomyces coelicolor pSCP1 plasmid). They could have several identical or very similar $a t t B$ sites as well, especially when the two copies are on the chromosome and a plasmid (Mycobacterium sp. MCS chromosome and pMKMS02 plasmid). Some plasmids also have copies of different variants (pREL1 from
Rhodococcus erythropolis PR4, pBD2 from R. erythropolis BD2; Table 2). At least some of these elements ought to be mobile since identical copies are found on chromosomes and plasmids and on different plasmids (identical copies at the nucleotide level in Mycobacterium sp. MCS chromosome and pMKMS02 plasmid, and pNL1 and pNL2 plasmids, respectively; data not shown).

Tn554 TnpC stimulates transposition and influences the orientation of transposed copies [34]. It may thus be dispensable, which could explain its absence from some of the related elements. Alternatively, unrelated proteins could be TnpC homologues although inspection of TnpB neighbors does not support this view.

\section{RIT elements: TBSSRs in trio}

Famints 1, 2 and 5 contain proteins that are encoded by three adjacent and overlapping genes, ritA, ritB and rit $C$. These TBSSR trios were first described in C. metallidurans CH34 [18]. Although not particularly well conserved, the three proteins in RITs make distinct families (RitA in Famint1, RitB in Famint5 and RitC in Famint2). They are particularly abundant in plasmid pHG1 from C. eutrophus H16. As shown in Table 1, all three families display a possible catalytic motif, suggesting that the three enzymes may be active, although it is still difficult to understand how a combination of three proteins would be needed to cleave four DNA strands in a breaking and rejoining reaction.

To access a larger and precompiled set of RIT TBSSRs, we again used the NCBI Protein Clusters, (Table 3 and Additional file 1: Table S4). As expected from the method used to assemble them, which is more stringent than our clustering procedure, these clusters are more granular, but nevertheless still clearly separate the A, B and C types of RIT encoded enzymes. To a few exceptions, these remain associated in trios of distinct clusters, with characteristic short overlaps between open reading frames (four to eight base pairs). Apparently, RITs are more frequent in chromosomes (in $62.3 \%$ of the cases) than in plasmids (in $37.7 \%$ of the cases). For 19 chromosomally-embedded RITs more information is available on the genomic context (through literature and Islandviewer [35]), indicating that for this group approximately $68 \%$ is located on a predicted genomic island.

In the absence of experimental results related to the mobility of the RIT structures, their distribution among different taxa and multiple copies in a genome provide some hints into this question. In particular, RITs with RitB in cluster CLSK923804 (group RIT7), which is associated with several types of RitA and RitC, are present in Firmicutes, $\alpha$-, $\beta$ - and $\delta$-proteobacteria (Additional file 1 : Table S4). Identical RIT copies are found in Burkholderia phytofirmans PsJN (three copies), Aromatoleum aromaticum EbN1 (three copies), Dinoroseobacter shibae DFL 
Table 2 Tn554-like elements in plasmids and chromosomes

\begin{tabular}{|c|c|c|c|c|c|c|}
\hline $\begin{array}{l}\text { Genbank } \\
\text { Acc. No. }\end{array}$ & Plasmids & sTBSSR CLSK No. & TnpB ID & LTBSSR CLSK No. & TnpA ID & TnpC \\
\hline NC_008270 & Rhodococcus sp. RHA1 pRHL2 & 636892 & 36 & 636891 & 41 & none \\
\hline NC_008697 & Nocardioides sp. JS614 pNOCA01 & 636892 & 36 & 636891 & 41 & none \\
\hline NC_008537 & Arthrobacter sp. FB24 plasmid 1 & 636892 & 36 & 636891 & 41 & none \\
\hline NC_003903 & Streptomyces coelicolor pSCP1 (2 copies) & 636892 & 36 & 636891 & 41 & none \\
\hline NC_007491 & Rhodococcus erythropolis PR4 pREL1 & 636892 & 36 & 636891 & 41 & none \\
\hline NP_898763 & R. erythropolis $\mathrm{pBD} 2$ & 636892 & 36 & 636891 & 41 & none \\
\hline NC_008271 & Rhodococcus sp. RHA1 pRHL3 & 523288 & 33 & 2316403 & 27 & 2316404 \\
\hline NC_007491 & R. erythropolis PR4 pREL1 & 2526550 & 33 & none & 27 & 2526549 \\
\hline NP_898763 & R. erythropolis pBD2 & 647580 & 33 & 647579 & 27 & 647578 \\
\hline NP_898763 & R. erythropolis pBD2 & 826662 & 33 & none & 27 & 647587 \\
\hline NC_005707 & Bacillus cereus pBc10987 & no cluster & 33 & 2462320 & 53 & 925958 \\
\hline NC_009426 & Novosphingobium aromaticivorans DSM 12444 pNL1 & 782394 & 62 & 782395 & 53 & 782393 \\
\hline NC_009427 & N. aromaticivorans DSM 12444 pNL2 (2 copies) & 782394 & 62 & 782395 & 53 & 782393 \\
\hline NC_009339 & Mycobacterium gilvum PYR-GCK pMFLV01 & no cluster & 33 & no cluster & 27 & no cluster \\
\hline NC_009339 & M. gilvum PYR-GCK pMFLV01 & 647579 & 33 & 730723 & 27 & 647580 \\
\hline NC_008147 & Mycobacterium sp. MCS plasmid 1 & 776375 & 33 & 776376 & 27 & 772808 \\
\hline NC_008704 & Mycobacterium sp. KMS pMKMSO2 & 776375 & 33 & 776376 & 27 & 772808 \\
\hline NC_004719 & Streptomyces avermitilis MA-4680 SAP1* & no cluster & 33 & none & - & none \\
\hline Genbank Acc. No. & Chromosomes & sTBSSR CLSK No. & TnpB ID & LTBSSR CLSK No. & TnpA ID & TnpC \\
\hline NC_008596 & Mycobacterium smegmatis str. MC2 155 & 776375 & - & 776376 & - & 772808 \\
\hline NC_008711 & Arthrobacter aurescens TC1 & 776375 & - & 776376 & - & 772808 \\
\hline NC_012803 & Micrococcus luteus NCTC 2665 (2 copies) & 776375 & - & 776376 & - & 772808 \\
\hline NC_008705 & Mycobacterium sp. KMS & 776375 & - & 776376 & - & 772808 \\
\hline NC_008726 & Mycobacterium vanbaalenii PYR-1 & 776375 & - & 776376 & - & 772808 \\
\hline NC_013235 & Nakamurella multipartita DSM 44233 (7 copies) & 647580 & - & 647579 & - & 647578 \\
\hline NC_010397 & Mycobacterium abscessus ATCC 19977 (2 copies) & 647580 & - & 647579 & - & 730718 \\
\hline NC_009338 & Mycobacterium gilvum PYR-GCK & 647580 & - & 647579 & - & 647578 \\
\hline NC_009077 & Mycobacterium sp. JLS & 647580 & - & 647579 & - & 730718 \\
\hline NC_008726 & M. vanbaalenii PYR-1 (4 copies) & 647580 & - & 647579 & - & 647578 \\
\hline NC_008726 & M. vanbaalenii PYR-1 & 647580 & - & 647579 & - & \\
\hline NC_008726 & M. vanbaalenii PYR-1 & 647580 & - & 647579 & - & none \\
\hline NC_008595 & Mycobacterium avium 104 & 647580 & - & 647579 & - & none \\
\hline NC_008268 & Rhodococcus jostii RHA1 & 647580 & - & 647579 & _- & none \\
\hline
\end{tabular}

CLSK No., Protein Cluster Database Number; ID, Famint number; none, there is no equivalent annotated protein at that position. no cluster, the protein is annotated but not part of a cluster. *, plasmid SAP1 has a truncated version of the Famint33 protein (246 aa only) and has no partners associated. -, not in the set of analyzed TBSSR proteins. S, shorter, L, longer.

12 (two copies), Heliobacterium modesticaldum Ice1 (two copies), Bordetella petrii DSM 12804 (two copies), Caulobacter sp. K31 (three copies), Mesorhizobium loti MAFF303099 (two copies) and Gramella forsetii KT0803 (two copies).

The RIT elements present in two strains of Acidithiobacillus ferrooxidans (ATCC 23720 and 53993), which are all from the same type (Additional file 1: Table S4 and Additional file 1: Table S5), are located in the transposase gene of a transposon related to Tn6049 from C. metallidurans $\mathrm{CH} 34$. This particular insertion site supports the mobility of this RIT, which is, however, tempered by the fact that these composite Tn::RIT structures are almost identical at the nucleotide sequence level and inserted at the same genomic location. The presence of a RIT6 insertion in the radC gene of Tn6054 (data not shown), a Tn4371-like ICE of C. metallidurans CH34 [17,18,20] also supports RIT mobility. Finally, on some plasmids (especially pHG1 from C. eutrophus and plasmid 2 from $A$. aromaticum sp. EbN1, Additional file 1: Table S4), RITs appear in complex combinations, where one or more of the RIT CDS is missing or truncated, again pointing towards some "mobility/ recombination" activity.

Since some strains contain two or more copies of the same RIT element, it was possible to deduce the length of the RIT to be around 3,500 bp. However, there seems to be some sequence variation at the ends of the element. Search for direct and inverted repeats in the sequence as 
Table 3 RIT elements classified according to NCBI Protein Clusters

\begin{tabular}{cccc}
\hline RIT type & $\begin{array}{c}\text { RitA } \\
\text { Famint1 }\end{array}$ & $\begin{array}{c}\text { RitB } \\
\text { Famint5 }\end{array}$ & $\begin{array}{c}\text { RitC } \\
\text { Famint2 }\end{array}$ \\
\hline RIT1 & CLSK521097 & CLSK2306416 & CLSK2306415 \\
RIT2 & CLSK2407259 & CLSK2314503 & CLSK458345 \\
RIT3A & CLSK382373 & CLSK747445 & CLSK502077 \\
RIT3B & CLSK2525360 & CLSK747445 & CLSK502077 \\
RIT3C & CLSK382373 & CLSK747445 & CLSK739704 \\
RIT4A & CLSK516123 & CLSK809014 & CLSK893868 \\
RIT4B & CLSK778800 & CLSK809014 & CLSK893868 \\
RIT5A & CLSK891968 & CLSK891969 & CLSK891970 \\
RIT5B & CLSK954479 & CLSK2468126 & CLSK891970 \\
RIT6 & CLSK953941 & CLSK953942 & CLSK953943 \\
RIT7A & CLSK2477616 & CLSK923804 & CLSK2477617 \\
RIT7B & CLSK962675 & CLSK923804 & CLSK2332435 \\
RIT7C & CLSK2809109 & CLSK923804 & CLSK2809110 \\
RIT8 & CLSK971115 & CLSK971116 & CLSK971117 \\
RIT9 & CLSK502016 & CLSK537353 & CLSK864421 \\
RIT10 & CLSK2491249 & CLSK2321302 & CLSK923805 \\
RIT11 & CLSK2471156 & CLSK962653 & CLSK962652 \\
\hline
\end{tabular}

RITs with the same number include at least one common protein cluster.

potential TBSSR binding sites did not produce any convincing result besides a 28-9 bp inverted repeat and overlapping palindromic sequence flanking ritA and ritC, respectively. These features do not fit well the usual multiple core and arm TBSSR binding sites found on, for example, temperate phage and ICE genomes.

Distribution of TBSSRs families among bacterial species and genera

To investigate the network of associations between bacterial groups given by the presence of different Famints, we linked pairs of the bacterial hosts based on the number of Famints they share (see Methods for details). The network (Figure 2) consists of six disjoint components. Five of these components are small and phylogenetically homogeneous. Two of these five harbor, respectively, five Firmicutes hosts and seven $\gamma$-proteobacteria, all of which are Enterobacteria. Interestingly, the latter separate from the rest of the proteobacterial hosts, which are found in the largest component. This may reflect the specialized habitat of Enterobacteria. In contrast, the largest component embraces hosts from different phyla, Proteobacteria, Actinobacteria, Acidobacteria and Bacteroidetes Two hubs mediate the inter-phyla and inter-class links, thus holding the component together. Erythrobacter and Acidiphilum connect $\alpha$ with $\beta$ - and $\gamma$-proteobacteria, and with Actinobacteria and Acidobacteria. Should these two hubs be removed, the component would split into two singletons and three clusters, two of which respectively include $\alpha$ - and $\gamma$-Proteobacteria. Nocardia, an Actinobacteria, makes a single connection with Agrobacterium in the $\alpha$-proteobacteria subgroup. The third cluster displays a heavy connectivity (number and weight of the links) and is phylogenetically very heterogeneous. It is composed of Frankia (Actinobacteria), Dinoroseobacter and Caulobacter ( $\gamma$-proteobacteria), Acidithiobacillus ( $\gamma$-proteobacteria), Gramella (Bacteroidetes), Solibacter (Acidobacteria). This network has to be taken cautiously, since the bias in the types of MGEs represented in the various hosts considered in our study cannot be neglected.

\section{Discussion}

The majority of tyrosine-based site-specific recombinases are specific to the type of mobile genetic element MCL clustering organized 27\% of the 1,309 TBSSR proteins encoded in plasmids, (pro)phages, GIs and ICEs into five "mixed" families, including the largest Famint0 of 210 members (that is, 16\%) that comprises all but one IntG proteins from GIs. Thus two-thirds of the TBSSR set $(63 \%)$ fit into families of four or more members that belong to the same type of MGE. Most families display a conserved potential catalytic motif (Table 1 ). Thus, by providing hints into the nature of the MGE type containing the protein, which can be further assessed by the function of neighboring genes on the genome of origin, the simple procedure used here could contribute greatly to improve the annotation of tyrosine-based site-specific recombinases.

A large proportion of the TBSSRs belonging to plasmids can still not be associated with any biological process. In particular, we could not unravel the families that promote the resolution of co-integrate and/or plasmid di-multi-mers. We did, however, identify families associated with new types of possible mobile elements.

First, a family of $S$. aureus Tn554-related transposons is present in Actinobacteria, with two adjacent TBSSRs coding genes. One of the proteins has a long N-terminal extension (over $400 \mathrm{aa}$ ) that displays no similarity with any obvious recognizable conserved protein domain and contains a potential C-terminal catalytic motif. Such a motif is also found on the shorter TBSSR in the tandems. In Tn554 both proteins are required for transposition [34], although their exact role in the reaction has not been established.

Second, the BIM associated TBSSR were so far found in a limited range of hosts all in the $\beta$-proteobacteria class, such that the mobility of BIMs presently lacks even theoretical support.

Third, the RIT elements are made of TBSSR trios and predominate in $\beta$ - and $\alpha$-proteobacteria. The distribution of different sub-types of RITs among these bacterial genera supports the hypothesis of their mobility, which nevertheless remains to be experimentally demonstrated. 


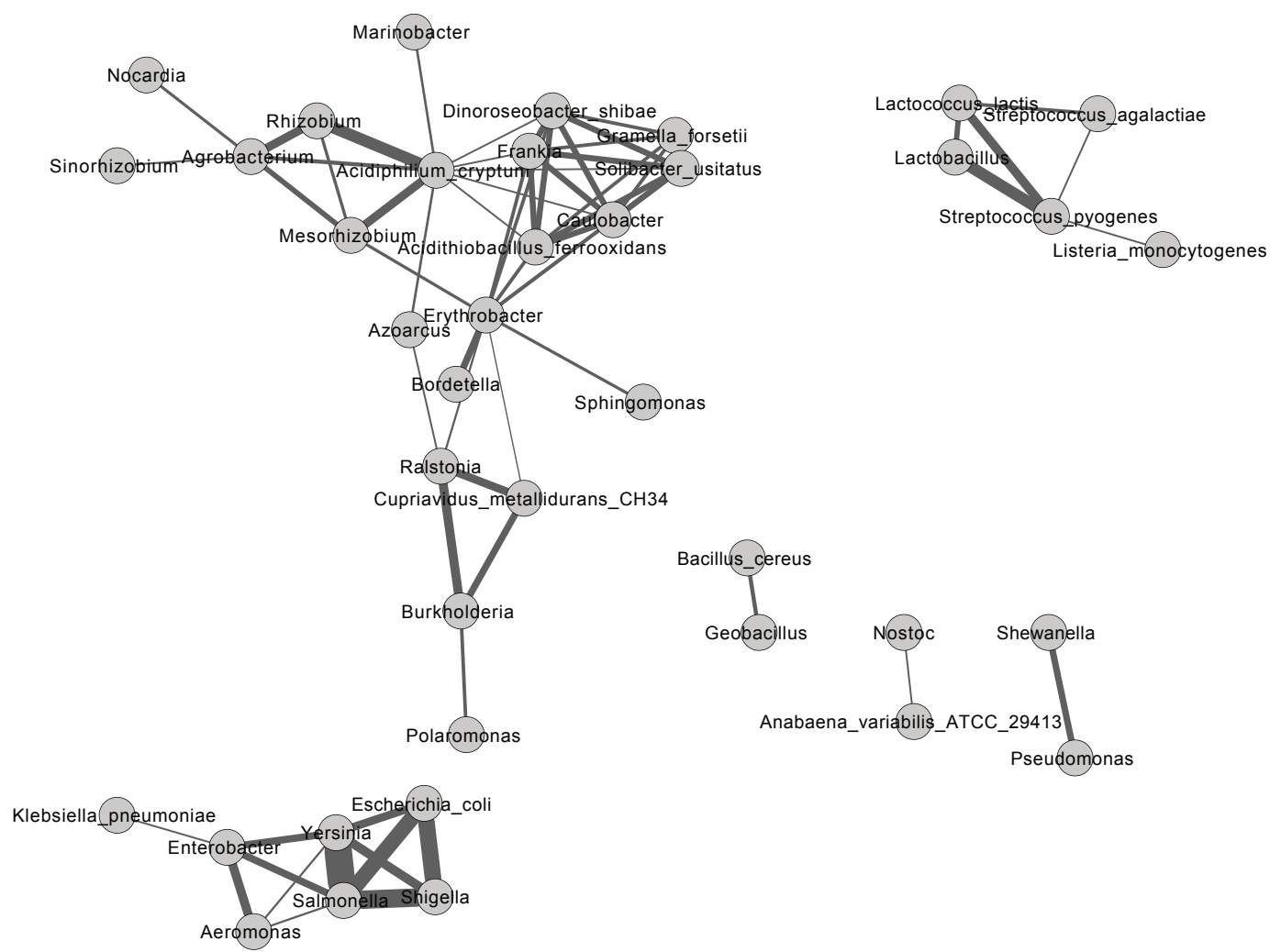

Figure 2 Weighted graphical representation of Famint families shared between bacterial hosts. Bacterial strains were grouped at the genus level unless there was a single representative at the strain or the species level. These groups of bacteria were represented in terms of the Famint families they contain. The graph was built as described in Methods. Nodes are bacterial genera, species or strains. They are linked by an edge if sharing Famint families. The thickness of the edges is proportional to the number of families shared by linked nodes. Note the tight grouping of Enterobacteria and Firmicutes.

\section{Improving the annotation of site specific and transpositional recombinases}

A more robust annotation of site specific and transpositional recombinases is desirable to avoid the propagation of, for example, 'phage-like integrase' annotation for a Tn4371-like ICE encoded TBSSR. We show above that with a limited set of TBSSRs of known origin, it becomes possible to infer the type of MGE coding for the enzyme in two-thirds of the cases. However, specifying the nature of the coding MGE is not sufficient to design a robust annotation of the protein. One way to go is to use a structured ontology, such as the GO ontology (http:// www.geneontology.org), which already offers a number of options that could be expanded. All TBSSRs belong to the same category of enzymes (molecular function in GO terminology), that is, "tyrosine-based site-specific recombinase activity" (GO:0009037) and catalyze the same biological processes "DNA integration" (GO:0015074) and "DNA excision" (GO:0044349) and should be annotated as such. When supported by the function of neighboring genes, the nature of the associated MGE sequence could be specified (using, for instance, the SO sequence ontology; http://www.sequenceontology.org/). In cases where the nature of the sequence provides a hint into a more precise biological process catalyzed by the TBSSR, these could as well be specified as "establishment as a prophage" or, "establishment as a GI", "resolution of plasmid dimers" or "resolution of cointegrates", using again defined terms as those offered by GO (or to be so but already available in the dedicated MeGO ontology http:// aclame.ulb.ac.be/Classification/mego.html).

As outlined in the introduction, TBSSRs catalyze the same biological processes as SBSSRs and DDE transposases/integrases, which also suffer from the absence of a coherent annotation. A similar approach to better discriminate these two other categories of proteins could be envisaged. The much higher sequence conservation of the SBSSRs may be a limitation, but there, enzymes that catalyze integration/excision reactions appear much larger (around 500 aa, [36]) than those that catalyze inversion reactions (200 aa). In the case of DDE transposases/ integrases, assembling a coherent set of sequences with indisputable MGE type origin is not straightforward, except for DDE enzymes encoded by IS sequences that 
are robustly classified in families available in the IS-Finder database (http://www-is.biotoul.fr/is.html, [37]). Nevertheless, considering separately the molecular function and the biological processes resulting from these enzyme activities would here again contribute to a more informative sequence annotation as in the case of TBSSRs.

\section{Conclusions}

A simple in silico procedure that uses a set of reference TBSSRs from defined MGE types combined with the analysis of the genetic context would allow for a greatly improved annotation of up to $60 \%$ of the tyrosine-based site-specific recombinases in prokaryotic genomes. In addition, the distribution among bacterial taxa of TBSSRs families should help to identify new types of mobile genetic entities deserving further experimental characterization.

\section{Methods}

A set of 1,309 TBSSR sequences (Additional file 1: Table S2) was assembled by grouping: i) 154 GI encoded proteins described by [13], ii) the proteins in all plasmid $(1,109)$, phage $(457)$ and predicted prophage $(760)$ protein families annotated as TBSSR (GO:0009037) in ACLAME DB version 0.4 [19], iii) a manually compiled list of proteins encoded by Tn4371-like transposons [20] and iv) proteins from RIT and BIM elements. The latter were identified by synteny using $C$. metallidurans genome sequence [18] on the MAGE annotation package [38] and the NCBI Protein Clusters [39]. A small number of identical proteins present in the ACLAME and additional lists, which are readily visible in the multiple alignments, were not sorted out. Protein sequences were clustered using the SSEARCH-MCL algorithm combination as follows: 1) Each sequence was used as a query sequence for SSEARCH to scan the set itself. The hits were limited to an E-value of $0.01 ; 2$ ) all the query-hit pairs + the $\log 10$ (E-value) obtained with SSEARCH were collected and provided as an input similarity matrix to the MCL algorithm; 3) MCL was executed with inflation factors (parameter influencing the clusters granularity) ranging from 1.2 (minimal value) up to 8.0 (maximum value) by steps of 0.2 , each giving a different sets of clusters. To select the optimal clustering, the homogeneity of the sets was assessed by calculating the intra-cluster clustering coefficient (ICCC, see [28] for the detailed procedure). Briefly, the ICCC measures the degree of inter-connectivity, as defined in the similarity matrix, of proteins within the same cluster. The inflation factor 1.8 gave the highest ICCC and was, therefore, selected as the optimal clustering result referred in this article as the protein families or Famints. The sequences from each cluster were provided to MUSCLE [40] using the default parameters to build the multiple sequence alignments. Each family was analyzed manually. Multiple alignments were visualized with the Jalview display and calculation package [41]. Putative catalytic sites were determined by visual inspection of the multiple alignments. Associations of protein families in ECMs [28] were retrieved from ACLAME version 0.4. Sharing of Famints families by hosts was analyzed using the Compare classes/clusters and Convert Graph methods provided on the NeAT web interface (http://rsat.bigre.ulb. ac.be/rsat/index_neat.html) [42], and the Cytoscape graph display [43].

\section{Additional material}

Additional file 1: Spreadsheet containing the supplementary data in the different sheets. Sheet TBSSR_plasmid_S1_Table S1. Number of

TBSSR genes in plasmid genomes in ACLAME version 0.4. BP: base pairs. Sheet Famints_S2 Table S2. TBSSR family (Famint) composition. Sheet BIM S3 Table S3. Identified BIM elements and associated NCBI Protein Clusters. SMC domain protein: predicted function of the second protein encoded by the element. Sheet RIT_S4 Table S4. Distribution of various RIT types among bacterial strains and composition of RIT types in terms of NCBI Protein Clusters. CDS distance is the distance in base pairs between the RitA and RitB (A-B) and RitB and RitC (B-C). Sheet Tn6049_RIT_S5 Table S5 Tn6049 related transposons in terms of NCBI Protein Cluster composition, some of which with an insertion of a RIT2 element. (XLS $256 \mathrm{~kb}$ ).

\section{Abbreviations}

aa: amino acid; BIM: bipartite module; ECM: Evolutionary Conserved Modules; GI(s): genomic island(s); ICCC: intra-cluster clustering coefficient; ICEs: integrated conjugative elements; MGE(s): mobile genetic element(s); MUSCLE: multiple sequence comparison by log-expectation; RIT: recombinase in trio; SBSSR(s): serine-based site specific recombinase(s); TBSSR (s): tyrosine-based site-specific recombinase(s)

\section{Acknowledgements}

This work was partly supported by the European Space Agency (ESAPRODEX) and the Belgian Science Policy (Belspo) through the MISSEX and COMICS projects, and by collaboration agreement between SCK.CEN and Université Libre de Bruxelles. GLM holds a postdoctoral fellowship from FWO (Fonds Wetenschappelijk Onderzoek)

\section{Author details}

'Unit of Microbiology (MIC), Belgian Nuclear Research Centre, SCK.CEN, Boeretang $200 \mathrm{Mol}$ B-2400, Belgium. ²Department of Informatics, Campus du Solbosch - CP197, 50 avenue F.D. Roosevelt, Bruxelles 1050, Belgium.

${ }^{3}$ Research Group of Bioinformatics and (Eco-)systems Biology, Department of Structural Biology, VIB, Pleinlaan 2, Brussels 1050, Belgium and Research Group of Bioinformatics and (Eco-)systems Biology, Microbiology Unit (MICR), Department of Applied Biological Sciences (DBIT), Vrije Universiteit Brussel, Pleinlaan 2, Brussels 1050, Belgium. ${ }^{4}$ Laboratoire Bioinformatique des Génomes et Réseaux (BiGRe), Université Libre de Bruxelles, Bvd du Triomphe, Bruxelles 1050, Belgium.

\section{Authors' contributions}

$\mathrm{RVH}$ and MM made the RIT and BIM analysis. RL maintains the ACLAME database and performed the computer procedures in this work. AT analyzed the Famint clusters, multiple alignments and NCBI Protein Clusters. GLM computed the graph in Figure 2. All the authors contributed to writing the manuscript.

\section{Competing interests}

The authors declare that they have no competing interests. 
Received: 9 October 2011 Accepted: 13 April 2012

Published: 13 April 2012

\section{References}

1. Jayaram M, Grainge I, Tribble G: Site-specific recombination by the Flp protein of Saccharomyces cerevisiae. In Mobile DNA II. Edited by: Craig NL, Cragie R, Gellert M, Lambowitz AM. Washington, DC: ASM Press; 2002:192-218.

2. Van Duyne GD: A structural view of tyrosine recombinase site-specific recombination. In Mobile DNA II. Edited by: Craig NL, Cragie R, Gellert M, Lambowitz AM. Washington, DC: ASM Press; 2002:93-117.

3. Rice PA: Theme and variation in tyrosine recombinases: structure of a Flp-DNA complex. In Mobile DNA II. Edited by: Craig NL, Cragie R, Gellert M, Lambowitz AM. Washington, DC: ASM Press; 2002:219-229.

4. Bouvier M, Demarre G, Mazel D: Integron cassette insertion: a recombination process involving a folded single strand substrate. EMBO J 2005, 24:4356-4367.

5. Dubois V, Debreyer C, Quentin C, Parissi V: In vitro recombination catalyzed by bacterial class 1 integron integrase Intl1 involves cooperative binding and specific oligomeric intermediates. PLOS One 2009, 4:e5228.

6. Dubois V, Debreyer C, Litvak S, Quentin C, Parissi V: A new in vitro strand transfer assay for monitoring bacterial class 1 integron recombinase Intl1 activity. PLoS One 2007, 2:e1315.

7. Espeli O, Marians KJ: Untangling intracellular DNA topology. Mol Microbiol 2004, 52:925-931.

8. Grainge I, Bregu M, Vazquez M, Sivanathan V, Ip SC, Sherratt DJ: Unlinking chromosome catenanes in vivo by site-specific recombination. EMBO J 2007, 26:4228-4238,

9. Sherratt DJ, Soballe B, Barre FX, Filipe S, Lau I, Massey T, Yates J: Recombination and chromosome segregation. Philos Trans $R$ Soc Lond $B$ Biol Sci 2004, 359:61-69.

10. Genka H, Nagata Y, Tsuda M: Site-specific recombination system encoded by toluene catabolic transposon Tn4651. J Bacteriol 2002, 184:4757-4766.

11. Hallet B, Vanhoof V, Cornet F: DNA site-specific resolution systems. In Plasmid Biology. Edited by: Funnell B, Phillips G. Washington, DC: ASM Press; 2004:145-179.

12. Leplae R, Lima-Mendez G, Toussaint A: A first global analysis of plasmid encoded proteins in the ACLAME database. FEMS Microbiol Rev 2006, 30:980-994.

13. Boyd EF, Almagro-Moreno S, Parent MA: Genomic islands are dynamic, ancient integrative elements in bacterial evolution. Trends Microbiol 2009, 17:47-53.

14. Ryan MP, Pembroke JT, Adley CC: Novel Tn4371-ICE like element in Ralstonia pickettii and genome mining for comparative elements. BMC Microbiol 2009, 9:242.

15. Austin S, Ziese M, Sternberg N: A novel role for site-specific recombination in maintenance of bacterial replicons. Cell 1981, 25:729-736.

16. Cambray G, Guerout AM, Mazel D: Integrons. Annu Rev Genet 2010, 44:141-166.

17. Toussaint A, Merlin C, Monchy S, Benotmane MA, Leplae R, Mergeay M, Springael D: The biphenyl- and 4-chlorobiphenyl-catabolic transposon Tn4371, a member of a new family of genomic islands related to IncP and Ti plasmids. Appl Environ Microbiol 2003, 69:4837-4845.

18. Van Houdt R, Monchy S, Leys N, Mergeay M: New mobile genetic elements in Cupriavidus metallidurans $\mathrm{CH} 34$, their possible roles and occurrence in other bacteria. Antonie Van Leeuwenhoek 2009, 96:205-226.

19. Leplae R, Lima-Mendez G, Toussaint A: ACLAME: a CLAssification of Mobile genetic Elements, update 2010. Nucleic Acids Res 2010, 38:D57-D61.

20. Van Houdt R, Toussaint A, Ryan MP, Pembroke JT, Mergeay M, Adley CC: The Tn4371 ICE family of bacterial mobile genetic elements. In Bacterial Integrative Mobile Genetic Elements. Edited by: Roberts AP, Mullany P. Austin, TX: Landes Bioscience; 2012: [http://www.ncbi.nlm.nih.gov/books/NBK63532/] (NCBI Bookshelf ID NBK63532)

21. Grindley ND, Whiteson KL, Rice PA: Mechanisms of site-specific recombination. Annu Rev Biochem 2006, 75:567-605.

22. Casjens S: Prophages and bacterial genomics: what have we learned so far? Mol Microbiol 2003, 49:277-300
23. Panis G, Mejean V, Ansaldi M: Control and regulation of KplE1 prophage site-specific recombination: a new recombination module analyzed. J Biol Chem 2007, 282:21798-21809.

24. Gyohda A, Furuya N, Ishiwa A, Zhu S, Komano T: Structure and function of the shufflon in plasmid R64. Adv Biophys 2004, 38:183-213.

25. Komano T, Fujitani S, Funayama N, Kanno A, Sakuma K: Physical and genetic analyses of Incl2 plasmid R721: evidence for the presence of shufflon. Plasmid 1990, 23:248-251.

26. Yoshida T, Furuya N, Ishikura M, Isobe T, Haino-Fukushima K, Ogawa T, Komano T: Purification and characterization of thin pili of Incl1 plasmids Collb-P9 and R64: formation of PilV-specific cell aggregates by type IV pili. J Bacteriol 1998, 180:2842-2848

27. Van Duyne GD: A structural view of Cre-loxP site-specific recombination. Annu Rev Biophys Biomol Struct 2001, 30:87-104.

28. Lima-Mendez G, Van Helden J, Toussaint A, Leplae R: Reticulate representation of evolutionary and functional relationships between phage genomes. Mol Biol Evol 2008, 25:762-777.

29. Hall RM: Mobile gene cassettes and integrons: moving antibiotic resistance genes in gram-negative bacteria. CIBA Found Symp 1997, 207:192-202, discussion 202-205.

30. Galimand M, Sabtcheva S, Courvalin P, Lambert T: Worldwide disseminated armA aminoglycoside resistance methylase gene is borne by composite transposon Tn1548. Antimicrob Agents Chemother 2005, 49:2949-2953.

31. Canton R, Coque TM: The CTX-M beta-lactamase pandemic. Curr Opin Microbiol 2006, 9:466-475.

32. Murphy E, Huwyler L, de Freire Bastos Mdo C: Transposon Tn554: complete nucleotide sequence and isolation of transposition-defective and antibiotic-sensitive mutants. EMBO J 1985, 4:3357-3365.

33. Murphy E, Reinheimer E, Huwyler L: Mutational analysis of att554, the target of the site-specific transposon Tn554. Plasmid 1991, 26:20-29.

34. Bastos MC, Murphy E: Transposon Tn554 encodes three products required for transposition. EMBO J 1988, 7:2935-2941.

35. Langille MG, Brinkman FS: IslandViewer: an integrated interface for computational identification and visualization of genomic islands. Bioinformatics 2009, 25:664-665

36. Smith MC, Brown WR, McEwan AR, Rowley PA: Site-specific recombination by phiC31 integrase and other large serine recombinases. Biochem Soc Trans 2010, 38:388-394.

37. Siguier P, Perochon J, Lestrade L, Mahillon J, Chandler M: ISfinder: the reference centre for bacterial insertion sequences. Nucleic Acids Res 2006, 34:D32-D36.

38. Vallenet D, Labarre L, Rouy Z, Barbe V, Bocs S, Cruveiller S, Lajus A, Pascal G, Scarpelli C, Medigue C: MaGe: a microbial genome annotation system supported by synteny results. Nucleic Acids Res 2006, 34:53-65.

39. Klimke W, Agarwala R, Badretdin A, Chetvernin S, Ciufo S, Fedorov B, Kiryutin B, O'Neill K, Resch W, Resenchuk S, Schafer S, Tolstoy I, Tatusova T: The National Center for Biotechnology Information's Protein Clusters Database. Nucleic Acids Res 2009, 37:D216-D223.

40. Edgar RC: MUSCLE: a multiple sequence alignment method with reduced time and space complexity. BMC Bioinforma 2004, 5:113.

41. Waterhouse AM, Procter JB, Martin DM, Clamp M, Barton GJ: Jalview Version 2 - a multiple sequence alignment editor and analysis workbench. Bioinformatics 2009, 25:1189-1191.

42. Brohee S, Faust K, Lima-Mendez G, Sand O, Janky R, Vanderstocken G, Deville $Y$, van Helden J: NeAT: a toolbox for the analysis of biological networks, clusters, classes and pathways. Nucleic Acids Res 2008, 36: W444-W451.

43. Smoot ME, Ono K, Ruscheinski J, Wang PL, Ideker T: Cytoscape 2.8: new features for data integration and network visualization. Bioinformatics 2011, 27:431-432.

doi:10.1186/1759-8753-3-6

Cite this article as: Van Houdt et al:: Towards a more accurate annotation of tyrosine-based site-specific recombinases in bacterial genomes. Mobile DNA 2012 3:6. 